\title{
Kraus representation of destruction of states for one qudit
}

\author{
Paweł Caban $*$ Kordian A. Smoliński $₫$ and Zbigniew Walczak \\ Department of Theoretical Physics, University of Łódź, Pomorska 149/153, 90-236 Łódź, Poland
}

(Dated: 29 September 2003)

\begin{abstract}
Quantum operations arise naturally in many fields of quantum information theory and quantum computing. One of the simplest example of quantum operation is the von Neumann-Lüders measurement. Destruction of states in quantum mechanics can be treated as a supplement to the von Neumann-Lüders measurement [P. Caban, J. Rembieliński, K. A. Smoliński and Z. Walczak, J. Phys. A 35, 3265 (2002)]. We show that destruction of states in one-qudit system is a quantum operation by finding its Kraus representation.
\end{abstract}

PACS numbers: 03.67. $-\mathrm{a}, 03.65 .-\mathrm{w}$

A general state change in quantum mechanics is described by the quantum operations formalism [1] (see also [2] and references therein) arising naturally in many fields of quantum information theory and quantum computing. In this formalism an input state $\rho$ of a system is connected with an output state $\mathcal{E}(\rho)$ by a linear, trace decreasing and completely positive map $\mathcal{E}$. Trace decreasing means that $\operatorname{tr}(\mathcal{E}(\rho)) \leqslant 1$ for all normalized density operators $\rho$. Complete positivity means that a map $\mathcal{E} \otimes \mathcal{I}$ is positive for all trivial extensions of a system (a system plus an ancilla). A quantum operation $\mathcal{E}$ can be represented (not uniquely) in a form known as the Kraus representation or the operator-sum representation

$$
\mathcal{E}(\rho)=\sum_{i} E_{i} \rho E_{i}^{\dagger}
$$

where the Kraus operators or operation elements $\left\{E_{i}\right\}$ satisfy the following condition

$$
\sum_{i} E_{i}^{\dagger} E_{i} \leqslant I
$$

Moreover, a map $\mathcal{E}$ which can be written in the form 11 is a quantum operation. If the Kraus representation contains only one operator we say that a quantum operation $\mathcal{E}$ is pure. The unitary evolution of density operator is an example of a pure quantum operation. Note that for a trace preserving operation the Kraus operators $\left\{E_{i}\right\}$ satisfy a completeness relation $\sum_{i} E_{i}^{\dagger} E_{i}=I$. The von Neumann-Lüders measurement with no selection is an example of a trace preserving quantum operation. In this paper, we show that a destruction of states [3] in a one-qudit system is a quantum operation by finding its Kraus representation.

Denote the Hilbert space of a system consisting of onequdit (a $d$-dimensional quantum system, with $d$ finite) by $\mathcal{H}^{1} \cong \mathbb{C}^{d}$. A state of the system is described by a density operator $\rho \in \operatorname{End}\left(\mathcal{H}^{1}\right)$, where $\operatorname{End}\left(\mathcal{H}^{1}\right)$ is the endomorphism space of $\mathcal{H}^{1}$. Such a description is not sufficient if a qudit can be destroyed by a measurement apparatus. This issue can be easily solved by introducing the vacuum state $|\mathrm{vac}\rangle$, which

\footnotetext{
*Electronic address: P.Caban@merlin.fic.uni.lodz.pl

${ }^{\dagger}$ Electronic address: K.A.Smolinski@merlin.fic.uni.lodz.pl

${ }^{\ddagger}$ Electronic address: Z.Walczak@merlin.fic.uni.lodz.pl
}

is orthogonal to any vector from $\mathcal{H}^{1}$ and for all observables $\hat{\Lambda}$ acting on $\mathcal{H}^{1}$ we adopt that $\hat{\Lambda} \mid$ vac $\rangle=0$. The vector $\mid$ vac $\rangle$ spans the one-dimensional vacuum space $\mathcal{H}^{0} \cong \mathbb{C}$. Therefore, the Hilbert space $\mathcal{H}$ of the system under consideration is the direct sum $\mathcal{H}^{1} \oplus \mathcal{H}^{0}$, and the states are mixtures of the elements from $\operatorname{End}\left(\mathcal{H}^{1}\right)$ and $\operatorname{End}\left(\mathcal{H}^{0}\right)$.

We now briefly review the destruction of states procedure introduced in Ref. [3]. Let $\hat{\Lambda}$ be an arbitrary observable on $\mathcal{H}^{1}$ with the spectrum $\Lambda$ and $\Omega$ be a subset of $\Lambda$. We denote the subspace spanned by all the eigenvectors corresponding to the eigenvalues from the subset $\Omega$ by $\mathcal{H}_{\Omega} \subset \mathcal{H}^{1}$ and the projector onto this subspace by $\Pi_{\Omega}$. If the qudit state is an element of $\operatorname{End}\left(\mathcal{H}_{\Omega}\right)$ then the qudit is destroyed, otherwise it is not. First, we measure with no selection the observable $\Pi_{\Omega}$. The density operator $\rho \in \operatorname{End}\left(\mathcal{H}^{1}\right) \oplus \operatorname{End}\left(\mathcal{H}^{0}\right)$ is reduced to $\Pi_{\Omega}^{\perp} \rho \Pi_{\Omega}^{\perp}+\Pi_{\Omega} \rho \Pi_{\Omega}$, where $\Pi_{\Omega}^{\perp}=\operatorname{id}_{\mathcal{H}}-\Pi_{\Omega}$. Then, after the destruction we get either the vacuum with the probability $\operatorname{tr}\left(\rho \Pi_{\Omega}\right)$ or the one-qudit state with the probability $\operatorname{tr}\left(\rho \Pi_{\Omega}^{\perp}\right)$. Therefore, the density operator is reduced to $\Pi_{\Omega}^{\perp} \rho \Pi_{\Omega}^{\perp}+\operatorname{tr}\left(\rho \Pi_{\Omega}\right)|\operatorname{vac}\rangle\langle\mathrm{vac}|$. Formally, the destruction with no selection in the set $\Omega$ of a one-qudit state $\rho$ is defined by the Kraus map $D_{\Omega}(\rho): \operatorname{End}\left(\mathcal{H}^{1}\right) \oplus \operatorname{End}\left(\mathcal{H}^{0}\right) \rightarrow \operatorname{End}\left(\mathcal{H}^{1}\right) \oplus$ $\operatorname{End}\left(\mathcal{H}^{0}\right)$, such that

$$
D_{\Omega}(\rho)=\Pi_{\Omega}^{\perp} \rho \Pi_{\Omega}^{\perp}+\widehat{\operatorname{tr}}\left(\Pi_{\Omega} \rho \Pi_{\Omega}\right),
$$

where the supertrace $\widehat{\operatorname{tr}}$ (we call $\widehat{\mathrm{tr}}$ a supertrace because it is a superoperator, i.e., it is the operator in the endomorphism space-see e.g., [4, [5]) is a linear map $\widehat{\operatorname{tr}}: \operatorname{End}(\mathcal{H}) \rightarrow$ $\operatorname{End}\left(\mathcal{H}^{0}\right)$ such that its action on the endomorphism of the form $|\chi\rangle\langle\phi| \in \operatorname{End}(\mathcal{H})$ is defined as follows

$$
\widehat{\operatorname{tr}}(|\chi\rangle\langle\phi|)=\langle\phi \mid \chi\rangle|\operatorname{vac}\rangle\langle\operatorname{vac}| .
$$

The supertrace is a quantum operation

$$
\begin{aligned}
\widehat{\operatorname{tr}}(|\chi\rangle\langle\phi|) & =\sum_{i=0}^{d}\langle\phi \mid i\rangle\langle i \mid \chi\rangle|\mathrm{vac}\rangle\langle\mathrm{vac}| \\
& =\sum_{i=0}^{d}|\mathrm{vac}\rangle\langle i \mid \chi\rangle\langle\phi \mid i\rangle\langle\mathrm{vac}|
\end{aligned}
$$

with operation elements $F_{i}=|\operatorname{vac}\rangle\langle i|$, where $\{|i\rangle\}_{i=0}^{d-1}$ is an orthonormal basis of $\mathcal{H}^{1}$, and $|d\rangle \equiv|\mathrm{vac}\rangle$. Note that the quantum operation (5) preserves the trace

$$
\sum_{i=0}^{d} F_{i}^{\dagger} F_{i}=\sum_{i=0}^{d}|i\rangle\langle\operatorname{vac} \mid \mathrm{vac}\rangle\left\langle i\left|=\sum_{i=0}^{d}\right| i\right\rangle\langle i|=\operatorname{id}_{\mathcal{H}} .
$$


For simplicity we assume that the basis in $\mathcal{H}_{\Omega}$ is a subset of $\{|i\rangle\}_{i=0}^{d-1}$. Therefore, $D_{\Omega}(\rho)$ may be written as

$$
D_{\Omega}(\rho)=\Pi_{\Omega}^{\perp} \rho \Pi_{\Omega}^{\perp^{\dagger}}+\sum_{|i\rangle \in \mathcal{H}_{\Omega}}\left(F_{i} \Pi_{\Omega}\right) \rho\left(F_{i} \Pi_{\Omega}\right)^{\dagger} .
$$

Thus, $D_{\Omega}$ is also a quantum operation with operation elements

$$
\begin{gathered}
E_{i}=F_{i} \Pi_{\Omega}, \quad i=0, \ldots, d, \\
E_{d+1}=\Pi_{\Omega}^{\dagger} .
\end{gathered}
$$

Note that $E_{i}=0$ for $|i\rangle \notin \mathcal{H}_{\Omega}$. Moreover, $D_{\Omega}$ is a trace preserving quantum operation

$$
\begin{aligned}
\sum_{k=0}^{d+1} E_{k}^{\dagger} E_{k} & =\Pi_{\Omega}^{\perp} \Pi_{\Omega}^{\perp}+\sum_{|i\rangle \in \mathcal{H}_{\Omega}} \Pi_{\Omega}^{\dagger} F_{i}^{\dagger} F_{i} \Pi_{\Omega} \\
& =\Pi_{\Omega}^{\perp}+\Pi_{\Omega}=\mathrm{id}_{\mathcal{H}} .
\end{aligned}
$$

As an example, consider destruction of states for one qubit $(d=2)$. Let $\{|0\rangle,|1\rangle\}$ be an orthonormal basis of $\mathcal{H}^{1}$. Assume that $\hat{\Lambda}|0\rangle=|0\rangle$ and $\hat{\Lambda}|1\rangle=-|1\rangle$, so $\Lambda=\{-1,1\}$. Therefore, we have the following cases:

(i) $\Omega=\{1\}$, the destruction with no selection takes place if the qubit is in the state $|0\rangle$. It means that $\Pi_{\Omega}=|0\rangle\langle 0|$ and $\Pi_{\Omega}^{\perp}=|1\rangle\langle 1|+| \mathrm{vac}\rangle\langle\mathrm{vac}|$. In this case operation elements for $D_{\Omega}$ are given by

$$
\begin{aligned}
& E_{0}=|\mathrm{vac}\rangle\langle 0|, \\
& E_{3}=|1\rangle\langle 1|+| \mathrm{vac}\rangle\langle\mathrm{vac}| .
\end{aligned}
$$

(ii) $\Omega=\{-1\}$, the destruction with no selection takes place if the qubit is in the state $|1\rangle$. It means that $\Pi_{\Omega}=|1\rangle\langle 1|$ and $\Pi_{\Omega}^{\perp}=|0\rangle\langle 0|+| \mathrm{vac}\rangle\langle\mathrm{vac}|$. In this case operation elements for $D_{\Omega}$ are given by

$$
\begin{aligned}
& E_{1}=|\operatorname{vac}\rangle\langle 1|, \\
& E_{3}=|0\rangle\langle 0|+| \mathrm{vac}\rangle\langle\mathrm{vac}| .
\end{aligned}
$$

(iii) $\Omega=\{1,-1\}$, the destruction with no selection takes place if the qubit is either in the state $|0\rangle$ or in the state $|1\rangle$. It means that $\Pi_{\Omega}=|0\rangle\langle 0|+| 1\rangle\langle 1|$ and $\Pi_{\Omega}^{\perp}=|v \operatorname{vac}\rangle\langle\mathrm{vac}|$. In this case operation elements for $D_{\Omega}$ are given by

$$
\begin{aligned}
& E_{0}=|\mathrm{vac}\rangle\langle 0|, \\
& E_{1}=|\mathrm{vac}\rangle\langle 1|, \\
& E_{3}=|\mathrm{vac}\rangle\langle\mathrm{vac}| .
\end{aligned}
$$

In conclusion, we have shown that destruction of states for one qudit is a trace preserving quantum operation by finding explicitly its Kraus representation. This result seems to be useful in the study of noisy quantum communication channels.

We are grateful to J. Rembieliński for useful discussions. This work was supported by University of Łódź and the Laboratory of Physical Bases of Processing of Information (LFPPI).
[1] K. Kraus, States, Effects, and Operations (Springer-Verlag, Berlin, 1983).

[2] M. A. Nielsen and I. L. Chuang, Quantum Computation and Quantum Information (Cambridge University Press, Cambridge, 2000).

[3] P. Caban, J. Rembieliński, K. A. Smoliński, and Z. Walczak, J.
Phys. A 35, 3265 (2002).

[4] J. Preskill, Physics 229: Advanced Mathematical Methods of Physics-Quantum Computation and Information, (http://www.theory.caltech.edu/people/preskill/ph229).

[5] C. M. Caves, J. Supercond. 12, 707 (1999). 